\title{
The parsec-scale structure, kinematics, and polarization of radio-loud narrow-line Seyfert 1 galaxies
}

\author{
J. L. Richards ${ }^{1, a}$, M. L. Lister ${ }^{1}$, T. Savolainen ${ }^{2}$, D. C. Homan ${ }^{3}$, \\ M. Kadler ${ }^{4}$, T. Hovatta ${ }^{5}$ A. C. S. Readhead ${ }^{6}$, T. G. Arshakian \\ and V. Chavushyan ${ }^{8}$ \\ ${ }^{1}$ Department of Physics and Astronomy, Purdue University, West Lafayette, Indiana, USA \\ ${ }^{a}$ email: jlr@purdue.edu \\ ${ }^{2}$ Max-Planck-Institut für Radioastronomie, Bonn, Germany \\ ${ }^{3}$ Department of Physics, Denison University, Granville, Ohio, USA \\ ${ }^{4}$ Institut für Theoretische Physik und Astrophysik, Universität Würzburg, Würzburg, \\ Germany \\ ${ }^{5}$ Aalto University Metsahövi Radio Observatory, Kylmälä, Finland \\ ${ }^{6}$ Department of Astronomy, California Institute of Technology, Pasadena, California, USA \\ ${ }^{7}$ I. Physikalisches Institut, Universität zu Köln, Köln, Germany \\ ${ }^{8}$ Instituto Nacional de Astrofísica, Óptica y Electrónica, Puebla, Mexico
}

\begin{abstract}
Several narrow-line Seyfert 1 galaxies (NLS1s) have now been detected in gamma rays, providing firm evidence that at least some of this class of active galactic nuclei (AGN) produce relativistic jets. The presence of jets in NLS1s is surprising, as these sources are typified by comparatively small black hole masses and near- or super-Eddington accretion rates. This challenges the current understanding of the conditions necessary for jet production. Comparing the properties of the jets in NLS1s with those in more familiar jetted systems is thus essential to improve jet production models. We present early results from our campaign to monitor the kinematics and polarization of the parsec-scale jets in a sample of 15 NLS1s through multifrequency observations with the Very Long Baseline Array. These observations are complemented by fast-cadence $15 \mathrm{GHz}$ monitoring with the Owens Valley Radio Observatory $40 \mathrm{~m}$ telescope and optical spectroscopic monitoring with with the $2 \mathrm{~m}$ class telescope at the Guillermo Haro Astrophysics Observatory in Cananea, Mexico.
\end{abstract}

Keywords. galaxies: individual (SDSS J095317.09+283601.4, SDSS J143509.49+313147.8, SDSS J095317.09+283601.4), galaxies: active, galaxies: Seyfert, galaxies: jets, radio continuum: galaxies

\section{Introduction}

The existence of jets in narrow-line Seyfert 1 galaxies (NLS1s) presents a challenge to our understanding of the relativistic jet phenomenon in active galactic nuclei (AGN). NLS1s are similar to ordinary Seyfert 1 galaxies but have unusually narrow broad lines $\left(\mathrm{FWHM}(\mathrm{H} \beta)<2000 \mathrm{~km} \mathrm{~s}^{-1}\right)$. NLS1s have many properties associated with weak or absent jets (e.g., Lacy et al. 2001; Véron-Cetty \& Véron 2001): they lie at the low end of the AGN black hole mass range $\left(10^{5}-10^{8} M_{\odot}\right.$; e.g., Zhou et al. 2006), accrete at a substantial fraction of the Eddington limit (e.g., Boller et al. 1996), and are likely found in spiral or disk host galaxies (Ohta et al. 2007). However, about 7\% are radio loud (Komossa et al. 2006) and among these, evidence of jets in these sources has been found, 
Table 1. Radio-Loud NLS1 monitoring sample (J2000 names).

\begin{tabular}{cccc}
\hline J0324+3410* & J0948+0022* & J1435+3131 & J1629+4007 \\
J0814+5609 & J0953+2836 & J1443+4725 & J1644+2619 \\
J0849+5108* & J1047+4725 & J1505+0326* & J1722+5654 \\
J0902+0443 & J1246+0238* & J1548+3511 & \\
Gamma-ray detection reported in Ackermann et al. (2011) or Foschini (2011).
\end{tabular}

including from the detection of $\mathrm{GeV}$ gamma-rays from several radio-loud NLS1s (e.g. Abdo et al. 2009). While a number of radio-loud NLS1s have been found to show jet morphologies on parsec scales (e.g., Doi et al. 2007; Gu \& Chen 2010; Doi et al. 2011), a comprehensive study of the properties of these jets has not been carried out. To address this, we are undertaking an observation campaign to characterize the parsec-scale radio jet morphologies and kinematics in a sample of radio-loud NLS1s.

Our observation program centers on a roughly year-long monitoring campaign with the Very Long Baseline Array (VLBA), augmented with fast-cadence single dish monitoring with the Owens Valley Radio Observatory $40 \mathrm{~m}$ telescope. Optical spectroscopic monitoring of several sources with the $2 \mathrm{~m}$-class telescope at the Guillermo Haro Optical Observatory (GHAO) was planned, but only one (J0324+3410) turned out to be bright enough. We do not discuss those results in these proceedings. Incidental to the VLBA program, we also made snapshot observations of three NLS1s with the Karl G. Jansky Very Large Array (VLA).

The sample, listed in Table 1, comprises the known radio-loud NLS1s above the celestial equator with a high-frequency $(1.4-15 \mathrm{GHz})$ archival flux density brighter than $30 \mathrm{mJy}$. These range in redshift from 0.061 to 0.799 , reaching the highest redshifts at which NLS1s can be identified. The sample includes all three northern-sky gamma-ray NLS1s in the 2-year Fermi Large Area Telescope (LAT) AGN Catalog (Ackermann et al. 2011) and several gamma-ray detections reported by Foschini (2011).

\section{Parsec-scale and single-dish monitoring}

The core of our program is the multifrequency, polarimetric monitoring with the VLBA. We will observe a total of 7 epochs with roughly 2 month separation, which will provide a total span of about a year. The first observations were in February 2014, and as of October we have completed four epochs. In each $24 \mathrm{~h}$ session, we interleave observations of our program sources and calibrators to provide broad $(u, v)$ coverage of each source. Each scan includes observations at 1.3, 2, 4, and $6 \mathrm{~cm}$, all in full polarization with 2-bit recording and $256 \mathrm{MHz}$ bandwidth in each polarization. This uses the full 2 Gbps data rate achievable with the VLBA digital back-end. Total intensity image noise levels of $0.07-0.1 \mathrm{mJy}$ beam $^{-1}$ are obtained in all bands. Phase referencing using nearby bright, compact targets is employed for the fainter sources in the program.

Currently, data reduction for the completed VLBA epochs is under way. Preliminary sample intensity maps are shown in Figure 1. All of the sources have been detected, and a variety of morphologies are found. Most resemble the compact, core-dominated structures commonly found in blazars (e.g., Lister \& Homan 2005). In all the resolved cases, the morphology is consistent with a one-sided core-jet structure. This resolved emission is found in 13 of the 15 sources, making these promising candidates for our planned kinematics studies.

In addition to the VLBA monitoring, all the sources in our sample are being monitored at $2 \mathrm{~cm}$ with the OVRO $40 \mathrm{~m}$ telescope as part of the ongoing blazar monitoring 

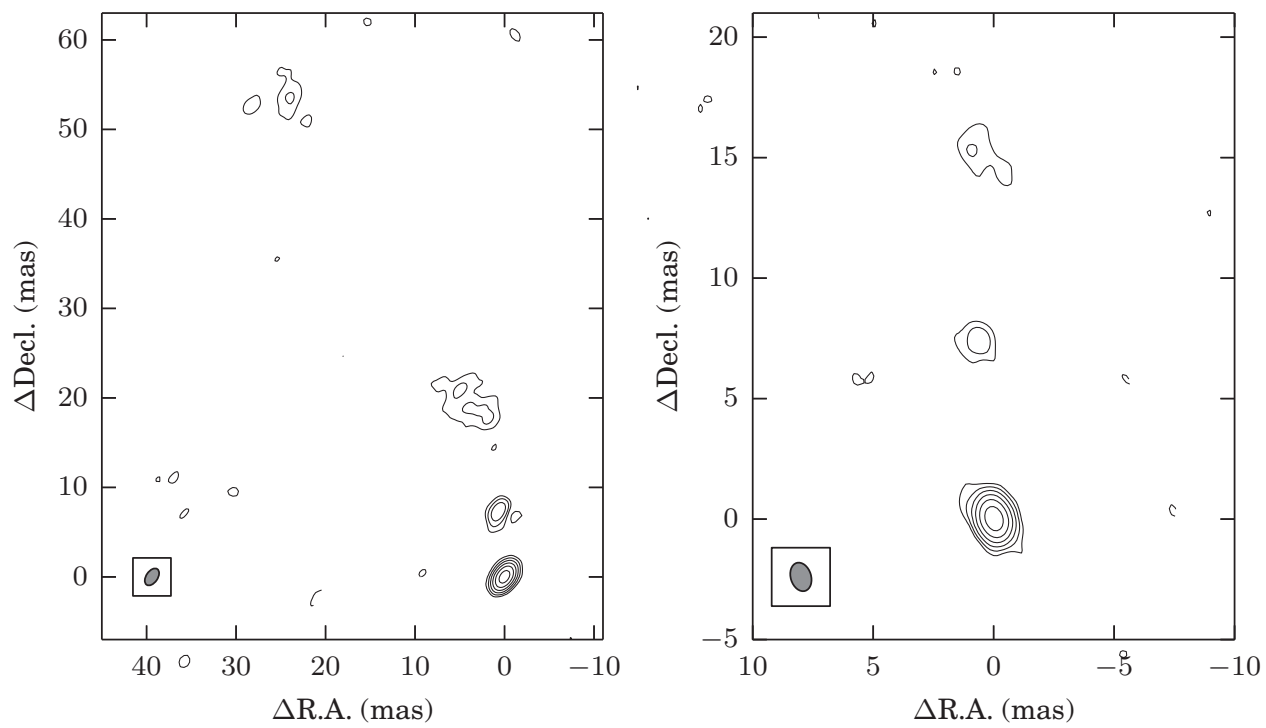

Figure 1. Preliminary VLBA total intensity maps of J1548+3511. Contours increase by factors of two. Left: $4 \mathrm{~cm}$ map from the first epoch (8 February 2014), showing emission extended to about 60 milli-arcsec. Right: $2 \mathrm{~cm}$ map from the third epoch (30 June 2014), showing good morphological agreement with the inner region of the $4 \mathrm{~cm}$ map.

program (Richards et al. 2011). These sources are observed about twice per week with a noise level of 3-5 mJy. We detect all our sources with mean flux densities between 11 and $480 \mathrm{mJy}$. Six of our 15 sources are too faint for variability studies. In five of the nine brighter sources we detect variability with amplitudes of $13-38 \%$, measured using the intrinsic modulation index introduced in Richards et al. (2011). This measure estimates the ratio of the standard deviation to the mean flux density after removing the effect of observational uncertainties. We find upper limits for the remaining four sources constraining them to be less than $5-20 \%$ variable. This range in variability amplitudes is comparable to that seen in blazars in the OVRO $40 \mathrm{~m}$ program.

\section{Observations of kiloparsec-scale extension}

At the outset of our program, extended radio emission on kiloparsec scales had been reported for only six radio-loud NLS1s (Doi et al. 2012). We observed three sources from our program for which the known radio positions were not precise enough for correlation of our VLBA data (J0953+2836, J1435+3131, and J1722+5654). These observations were carried out at $9 \mathrm{GHz}$ while the VLA was being reconfigured from B to A array, its most extended and therefore highest resolution configuration. The restoring beam for these observations was about 0.2 arcsec. This provided more than an order of magnitude increase in resolution compared to most previous NLS1 observations. In 10 min integrations with $2 \mathrm{GHz}$ total bandwidth, we reached a noise level of $6-8 \mu \mathrm{Jy} \mathrm{beam}^{-1}$ in the naturally weighted maps.

We detected significant kiloparsec-scale extended emission in all three sources. All three showed core-dominated morphologies with prominent two-sided edge-brightened lobes reminiscent of FR II radio galaxies. The full extents of the sources ranged from 410 arcsec, corresponding to projected lengths of $23-70 \mathrm{kpc}$. Our observations, which will 
be presented in detail in a forthcoming publication, increase the number of radio-loud NLS1s with kiloparsec-scale extensions by $50 \%$, from six to nine.

\section{Discussion}

The preliminary results of our observations provide further strong evidence for the presence of relativistic jets in radio-loud NLS1s. The one-sided morphologies we find on parsec-scales are strongly reminiscent of those found in much larger, more powerful blazars. These morphologies are consistent with the standard picture of a pair of oppositely directed relativistic jets escaping from near the central black hole. The morphologies on kiloparsec scales are also consistent with this picture, showing that the parsec-scale jets continue out to larger scales. Although we have only observed three sources with the VLA, all three show kiloparsec-scale extension. This suggests that, in contrast to previous findings (Ulvestad et al. 1995), when observed with the enhanced sensitivity of the recently upgraded VLA, such extended emission is common among the sources in our sample. In our OVRO single-dish monitoring we find variability similar to that seen in many blazars, furthering the comparison. It thus seems that although they are an order of magnitude or more smaller than typical blazars and radio galaxies, radio-loud NLS1s nonetheless frequently produce radio jets that extend to kiloparsec scales. Our monitoring program will soon enable a more detailed comparison of the jets in these classes of sources, including their polarization and kinematic behavior.

\section{Acknowledgements}

This work was funded by the National Aeronautics and Space Administration through Fermi Guest Investigator grant NNX13AO79G. The National Radio Astronomy Observatory is a facility of the National Science Foundation operated under cooperative agreement by Associated Universities, Inc. This research has made use of the NASA/IPAC Extragalactic Database (NED) which is operated by the Jet Propulsion Laboratory, California Institute of Technology, under contract with the National Aeronautics and Space Administration.

\section{References}

Abdo, A. A., et al. 2009, ApJ, 707, L142

Ackermann, M., et al. 2011, ApJ, 743, 171

Boller, T., Brandt, W. N., \& Fink, H. 1996, A\&A A, 305, 53

Doi, A., Asada, K., \& Nagai, H. 2011, ApJ, 738, 126

Doi, A., Nagira, H., Kawakatu, N., Kino, M., Nagai, H., \& Asada, K. 2012, ApJ, 760, 41

Doi, A., et al. 2007, PASJ, 59, 703

Foschini, L. 2011, in Proceedings of Science, Vol. NLS1, Narrow-Line Seyfert 1 Galaxies and their Place in the Universe, L. Foschini, et al. (Eds.), id. 24

Gu, M. \& Chen, Y. 2010, AJ, 139, 2612

Komossa, S., Voges, W., Xu, D., et al. 2006, AJ, 132, 531

Lacy, M., Laurent-Muehleisen, S. A., Ridgway, S. E., et al. 2001, ApJ, 551, L17

Lister, M. L. \& Homan, D. C. 2005, AJ, 130, 1389

Ohta, K., Aoki, K., Kawaguchi, T., \& Kiuchi, G. 2007, ApJS, 169, 1

Richards, J. L., et al. 2011, ApJS, 194, 29

Ulvestad, J. S., Antonucci, R. R. J., \& Goodrich, R. W. 1995, AJ, 109, 81

Véron-Cetty, M. P. \& Véron, P. 2001, A\& A, 375, 791

Zhou, H., Wang, T., Yuan, W., Lu, H., Dong, X., Wang, J., \& Lu, Y. 2006, ApJS, 166, 128 